\title{
Caveolin-1 is involved in high glucose accelerated human glomerular mesangial cell senescence
}

\author{
Xin Feng ${ }^{1}$, Wei Gao ${ }^{1}$, and Yao $\mathrm{Li}^{2}$
}

${ }^{1}$ Department of Rheumatology, The First Affiliated Hospital of Liaoning Medical University, Jinzhou; ${ }^{2}$ Department of Physiology, Liaoning Medical University, Jinzhou, China

Received: August 1, 2015

Revised : September 7, 2015

Accepted: October 15, 2015

\section{Correspondence to}

Wei Gao, M.D.

Department of Rheumatology, The First Affiliated Hospital of Liaoning Medical University, No 2, RenMin Street, Guta, Jinzhou 121000, China

E-mail: xinxinyiran@126.com
Background/Aims: We demonstrated the role of caveolin-1 involved in high glucose (HG)-induced glomerular mesangial cells (GMCs) senescence.

Methods: HG was used to stimulate GMCs. The telomere lengths were analyzed by Southern blot. $\beta$-Galactosidase staining was determined. The expressions of caveolin-1 and $\mathrm{P}_{53}$ proteins were determined by Western blot.

Results: Treatment with high concentrations of glucose induced GMC senescence accompanied by shortened telomere length and increase of $\beta$-galactosidase staining as well as $\mathrm{P}_{53}$ protein, which was abrogated after application of caveolin-1siRNA.

Conclusions: This study proved that HG induced cell senescence in GMCs. The caveolin-1 is involved in HG-induced mesangial cell senescence, and blocking caveolin-1 significantly reduced cell senescence. The effect of caveolin-1 is mediated by $\mathrm{P}_{53}$ pathway.

Keywords: Mesangial cells; Aging; Caveolin-1

\section{INTRODUCTION}

Aging is the gradual changes in the structure and function of human tissues under internal and external environmental pressure. The kidney is most likely to be affected by aging [1]. Many reviews have described the overall function of the aging kidney [2], but at present the factors influencing aging of the kidney are not fully elucidated. Glomerular mesangial cells (GMCs) are most important cells. GMCs can regulate filtration surface area and have the function of secreting cell matrix, producing cell factor, removing the large molecules. The changes in GMCs phenotype and function play important roles in the aging of the kidney.

Cell senescence is characterized by an growth arrest and functional and morphological changes [3], including increased expression of senescence markers, such as senescence-associated-galactosidase (SA- $\beta$-gal), telomere shortening and negative regulators of the cell cy- cle [4]. Cellular senescence can be induced by a lot of stresses such as oxidative stressors or inflammation. Increasing evidence indicates that cells has a shortened proliferative lifespan and enter the state of senescence earlier after exposed to high glucose (HG) [5]. However, the underlying mechanism is not clear.

Caveolae are a special type of lipid rafts coated with caveolin proteins, which can regulate the activity of cell signaling receptors and contribute to lipid transport [6]. Caveolin-1 is the main component of caveolae, which is involved in the regulation of many cellular processes, including growth, migration, and senescence $[7,8]$. Recently, some studies results have indicated the role of caveolin-1 in stress-induced premature cellular senescence. Caveolin-mediated signaling may contribute to explain, at the molecular level, how oxidative stress promotes cell senescence [9].

In this study, we test expression of caveolin-1 in the aging human mesangial cells induced by HG. We further 
study the expression of $\mathrm{P}_{53}$ protein and $\beta$-galactosidase as well as telomere length after application of caveolin-1 silencing vectors. The results of this project can define the effect and mechanism of caveolin-1 on aging human mesangial cells induced by HG and provide a new target for the treatment of renal failure.

\section{METHODS}

\section{Materials}

The human GMCs and mesangial cell medium (4201 cell medium) were purchased from ScienCell Research Laboratories (Carlsbad, CA, USA), and a telomere length determination kit was obtained from Roche Applied Sciences (Penzberg, Germany). The antibodies against caveolin-1 and $\mathrm{P}_{53}$ were purchased from Abcam Technology (Cambridge, UK).

\section{Cell culture}

GMCs were cultured in 4201 cell medium (glucose, 5 $\mathrm{mmol} / \mathrm{L}$ ) supplemented with $10 \%$ fetal bovine serum (FBS) in an atmosphere of $5 \% \mathrm{CO}_{2}$ at $37^{\circ} \mathrm{C}$. Cells were dispersed with $0.25 \%$ trypsin and subcultured at a ratio of $1: 2$ when grown to $80 \%$ to $90 \%$ confluence in $25-\mathrm{cm}^{2} \mathrm{cul}-$ ture flasks. Cells between five and eight passages were used. For the experiments, cells were synchronized by culturing in serum free medium for 24 hours to ensure Go arrest. After synchronized, glucose (30 $\mathrm{mmol} / \mathrm{L}$ ) was added to the culture medium, and cells were stimulated for 96 hours. Experimental GMCs were divided into groups of: HG + transfected caveolin-1 small interfering RNA cells (HG-siRNA), transfected siRNA negative control cells (HG-NC), HG group cells and control group cells.

\section{Cell morphology}

GMCs were washed twice with phosphate-buffered saline (PBS). Morphologic changes in cells were evaluated under the microscope.

\section{$\beta$-Galactosidase staining}

GMCs were washed three times with PBS when grown into $80 \%$ confluence. The fixation solution which included $2 \%$ formaldehyde, $0.2 \%$ glutaraldehyde was added to cells at room temperature. After 5 minutes, GMCs were washed three times with PBS. Then, freshly prepared $1 \mathrm{~mL}$ SA- $\beta$-gal was used to stain the cells at $37^{\circ} \mathrm{C}$ for 12 to 16 hours. The blue precipitates can be seen in the cytoplasm. For each sample, six fields were randomly selected. The percentage of positive cells was calculated.

\section{Detection of reactive oxygen species in cells}

Dichlorodihydrofluorescein diacetate (DCFH-DA, 10 $\mu \mathrm{mol} / \mathrm{L}$ ) was used as probe. Cells were centrifuged and collected. GMCs were washed three times with PBS. DCFH-DA was added to the cells at 37 minutes for 10 minutes. Flow cytometry was used to detect the average fluorescent intensity of intracellular dendritic cell. Excitation light was $488 \mathrm{~nm}$.

\section{Southern blot analysis}

Genomic DNA was taken using a cell genomic DNA extraction kit (Gentra, Minneapolis, MN, USA). Restriction enzyme Hinf I/Rsa I was used to digest the DNA sample overnight at $37^{\circ} \mathrm{C}$. The digested fragments were got and separated with $0.8 \%$ agarose gel electrophoresis. After electrophoresis, the gel was immersed in $0.25 \mathrm{M} \mathrm{HCl}$ for 30 minutes and denatured in $0.4 \mathrm{M} \mathrm{NaOH}$ and 1.5 $\mathrm{M} \mathrm{NaCl}$ for 30 minutes. The DNA was transferred onto a positively charged nylon membrane. The membrane was prehybridized in hybridization buffer at $42^{\circ} \mathrm{C}$ for 1 hour and then hybridized with a telomere probe in hybridization buffer at $42^{\circ} \mathrm{C}$ for 3 hours. After washed twice with washing solution, the membrane was rinsed with the blocking reagent. The hybridized probe was detected by chemiluminescence. The membrane was exposed to Hyperfilm. The average telomere terminal restriction fragment (TRF) length was calculated using the formula: $\mathrm{TRF}=\Sigma(\mathrm{ODi}) / \Sigma(\mathrm{ODi} / \mathrm{Li})$, where ODi is the integrated signal intensity and $\mathrm{Li}$ is the DNA length at position.

\section{Western blot analysis}

GMCs were prepared after stimulation. GMCs were lysed with radioimmunoprecipitation assay solution and centrifugated under $12,000 \times g$ at $4^{\circ} \mathrm{C}$ for 5 minutes. The supernate was collected, and protein concentration was evaluated by bicinchoninic acid assay. The concentrations of protein samples were equal. After separated by $8 \%$ SDS-polyacrylamide gel electrophoresis $(80 \mathrm{~V}, 1$ hour), protein were electrically transferred onto the nitrocellulose membrane (6o V, 3 hours). 
The membrane was blocked with $5 \%$ non-fat dry milk for 2 hours, and incubated overnight with caveolin-1, $\mathrm{P}_{53}$ antibodies. Then, the membrane was incubated with secondary antibodies at room temperature for 2 hours. Color was developed using the alkaline phosphatase approach. A quantitative analysis of grey intensity was carried out with Scion Corp. (Frederick, MD, USA).

\section{siRNA synthesis}

Single siRNA strands were 5'-GCAGUUGUACCAUGCAUUAT'T-3'(sense)and 5'-UAAUGCAUGGUACAACUGCT'T-3'(antisense). Single siRNA NC strands were 5'-UUCUCCGAACGUGUCACGUTT-3'(sense) and 5'-ACGUGACACGUUCGGAGAATT-3'(antisense). All single siRNA strands were synthesized at Invitrogen (Waltham, MA, USA).

\section{siRNA transfection}

The lipofectamine 2000 (Invitrogen) were used to transfect the GMCs according to the manufacturer's instruction. Briefly, $2 \times 10^{5}$ GMCs were cultured in six-well plates in $2 \mathrm{~mL}$ of antibiotic-free growth medium supplemented with FBS for 24 hours. The supernatant was removed before transfecting. These $100 \mathrm{pM}$ of siRNA, 5 $\mu \mathrm{L}$ of transfection reagent, and $500 \mu \mathrm{L}$ serum-free mesangial cell medium were mixed, thoroughly, and incubated at room temperature for 20 minutes. Mixture was then added to cells and incubated for another 6 hours.

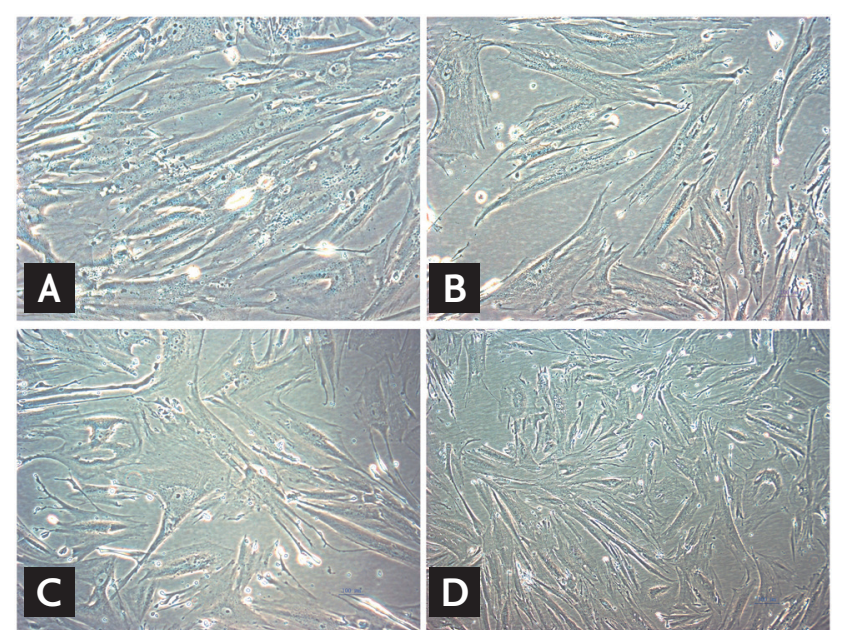

Figure 1. Morphologic changes in cells. (A) Control group, (B) high glucose (HG)-group, (C) HG-negative control group, and (D) HG-siRNA group cells captured under an optical microscope (×100). Typical results of three independent experiments are shown.
Supernatant was then removed and replaced with fresh media containing 10\% FBS. After 48 hours incubation, cells were harvested for further analysis. Efficiency of the transfection was assessed by fluorescence microscopy.

\section{Statistical analysis}

The experimental data were presented as means with standard deviations (SD) and analyzed with SPSS version 19.0 (IBM Co., Armonk, NY, USA). The intergroup data were compared via analysis of variance, a significance level of 0.05 was used.

\section{RESULTS}

\section{Cultured GMCs show accelerated senescence when exposed to HG media}

We evaluated morphologic alterations in the GMCs by optical electron microscopy. The cells in the control group displayed well-arranged spindle morphology, with more dividing cells and cell borders were clear. The cells in the HG group exhibited large sizes. Abundant particles and vacuoles could be seen in the cytoplasm. The number of cells decreased (Fig. 1A and 1B). Next, we determined whether the replicative arrest was associated with senescence by SA- $\beta$-gal staining. After treatment with HG for 96 hours, SA- $\beta$-gal staining in the GMCs of the HG group $(88.31 \% \pm 5.69 \%)$ was significantly increased compared with the control cells $(12.11 \% \pm 2.54 \%$, $p<0.05$ ) (Fig. 2A and 2B). We used Southern blot analysis to investigate the effect of HG on TRF lengths in GMCs. In the control group, TRF lengths were $5.63 \pm 0.046 \mathrm{~kb}$, HG-grown GMCs had lower TRF lengths $(3.16 \pm 0.087$ $\mathrm{kb})$ than the control group. These results revealed that telomere DNA in GMCs lost significantly after 96 hours treatment with HG (Fig. 3).

\section{The level of reactive oxygen species in the GMCs}

The results showed that the level of reactive oxygen species (ROS) in the GMCs of the HG group was increased compared with the control cells $(442.3 \% \pm 36.2 \%$ and $188.11 \% \pm 5.23 \%, p<0.05$ ), Compared with the HG group, the level of ROS was decreased in the HG-siRNA group $(442.3 \% \pm 36.2 \%$ and $235.76 \% \pm 8.31 \%, p<0.05)$. 

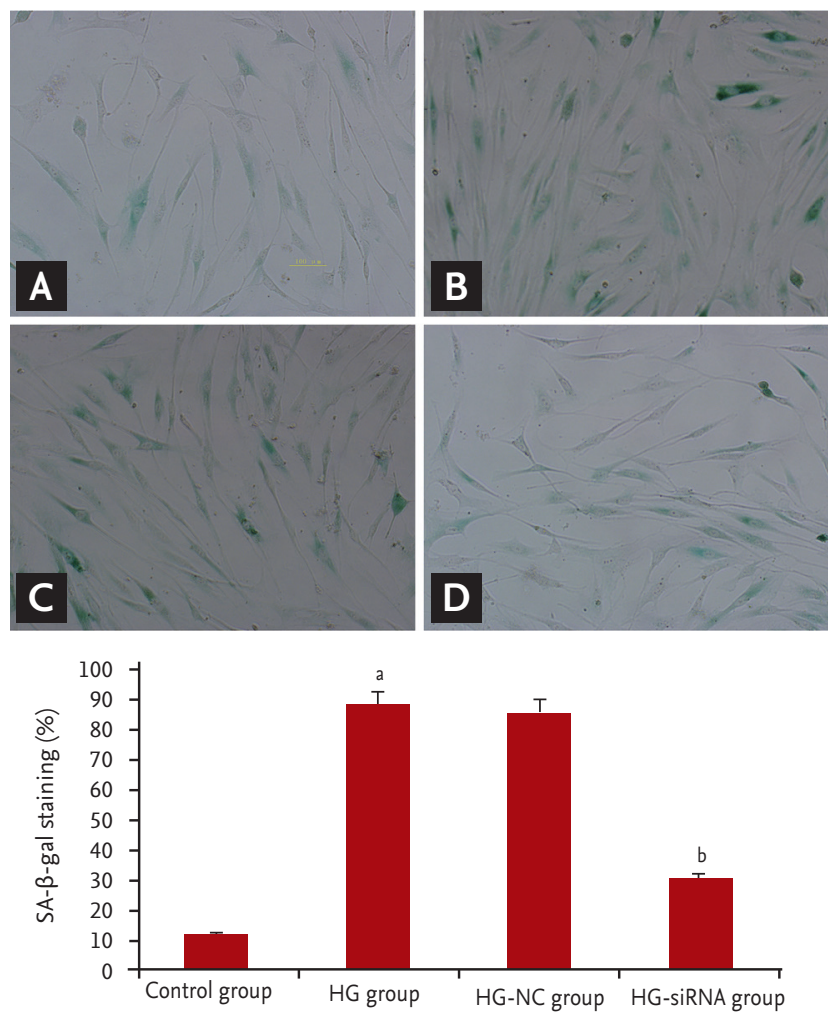

Figure 2. Senescence-associated-galactosidase (SA- $\beta$-gal) staining in glomerular mesangial cells. (A) Control group, (B) high glucose (HG)-group, (C) HG-negative control (NC) group, and (D) HG-siRNA group cells (×200). Results are representative of two regions from three independent experiments. ${ }^{\mathrm{a}} \mathrm{p}<0.05$ vs. control group, ${ }^{\mathrm{b}} \mathrm{p}<0.05$ vs. HG group.

\section{Increase in caveolin-1 during aging}

To determine changes in caveolin-1 during the process of GMC senescence stimulated by HG, Western blot analysis was performed. The results showed that compared with the control group, the expression of caveolin-1 protein was significantly elevated in the HG group $(p<0.05)$ (Fig. 4).

\section{The inhibition efficiency of transfection}

We evaluated the inhibition efficiency of transfection in the GMCs by fluorescence microscope. Green fluorescence could be seen in the cells which were successfully transfected. The inhibition efficiency was determined by reverse transcription polymerase chain reaction. After 24 hours, the expression of caveolin-1 was inhibited. Compared with HG group, the expression level of caveolin-1 was $63 \% \pm 9 \%$ after 48 hours and $12 \% \pm 4 \%$ after 72 hours in HG-siRNA group. But the expression level of caveolin-1 in HG-NC group was not significantly differ-
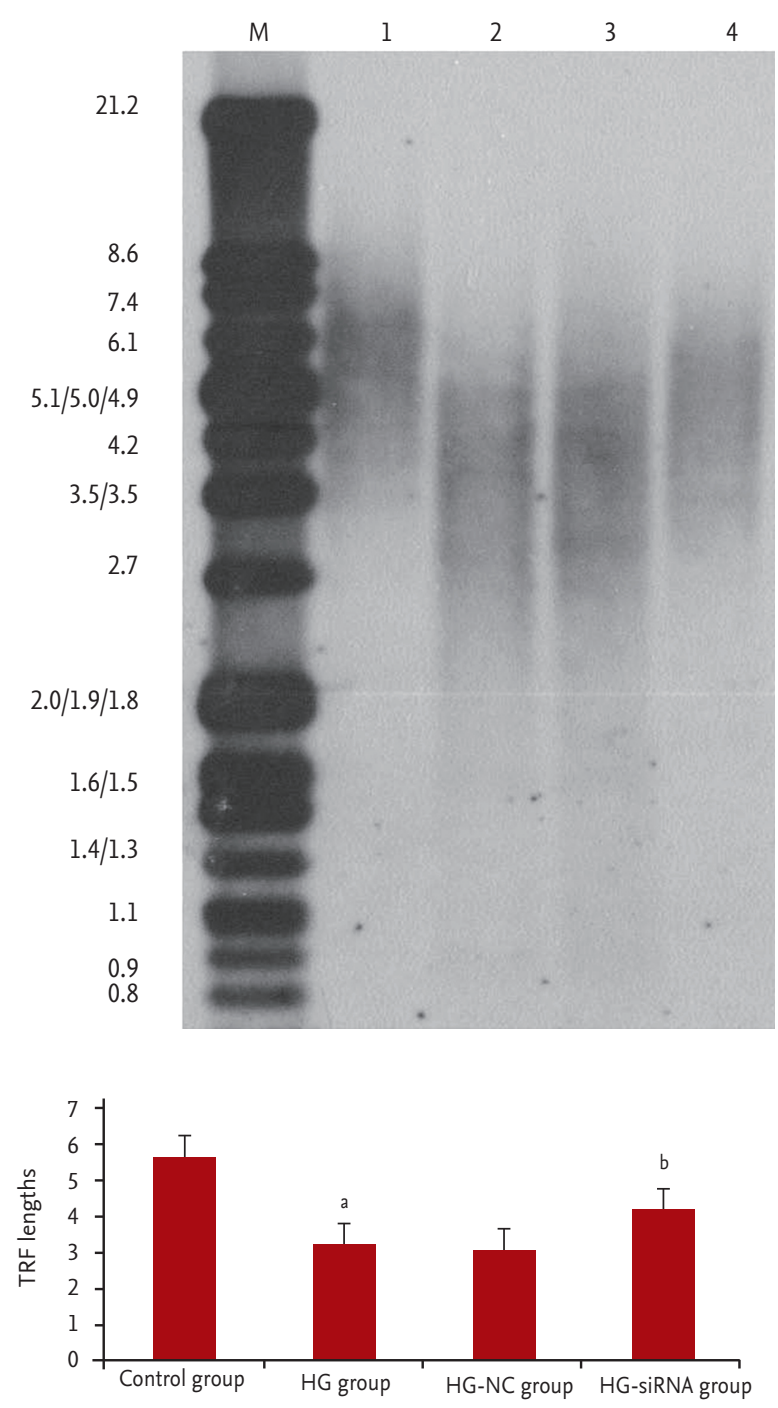

Figure 3. Average telomere length (terminal restriction fragment [TRF]) in the cells were measured by Southern blots: lane 1, control group; lane 2, high glucose (HG)-group; lane 3, HG-negative control (NC) group; and lane 4, HG-siRNA group cells. Typical results of three independent experiments are shown. ${ }^{a} p<0.05$ vs. control group, ${ }^{b} p<0.05$ vs. HG group.

ent between $0,24,48$, and 72 hours, the difference was not statistically significant. The expression of caveolin-1 protein of HG-siRNA group was significantly decreased than HG-NC group.

\section{Function of caveolin-1 in delaying GMCs aging}

Compared with HG-group and HG-NC-group, morphologic changes were improved in the HG-siRNA group. Optical microscopy revealed HG-siRNA group cells to be 
regularly aligned, with nuclear membrane invagination (Fig. 1B-1D). Compared with the HG group and HG-NCgroup, SA- $\beta$-gal staining was decreased in the HG-siR-
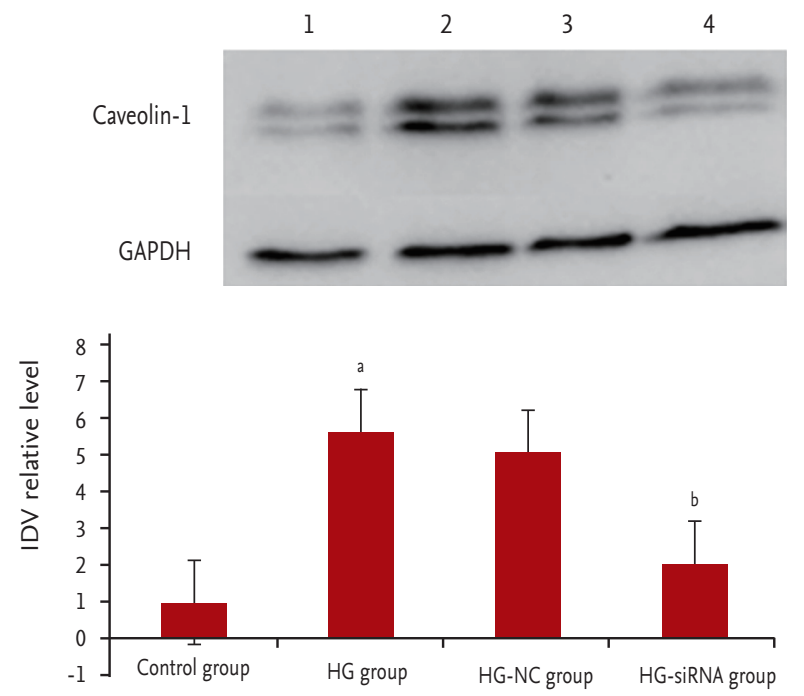

Figure 4. Western blot analysis. Lane 1, control group; lane 2, high glucose (HG)-group; lane 3, HG-negative control (NC) group; and lane 4, HG-siRNA group cells. Integrated density values (IDVs) of caveolin-1 are shown. GAPDH, glyceraldehyde 3-phosphate dehydrogenase. ${ }^{a} p<0.05$ vs. control group, ${ }^{b} p<0.05$ vs. HG group.

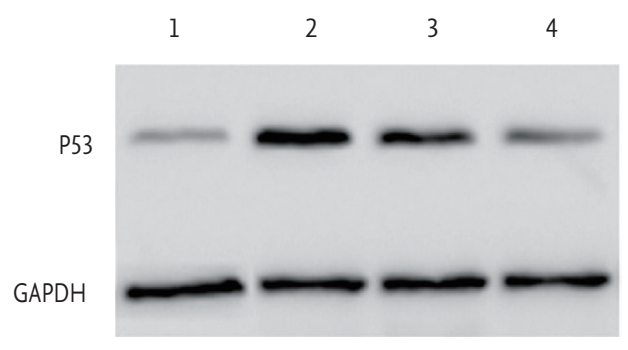

A

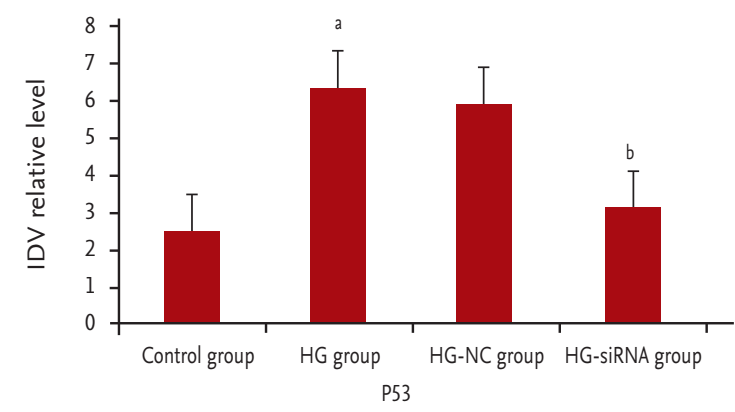

NA group $(30.73 \% \pm 3.23 \%, 88.31 \% \pm 5.69 \%$, and $85.56 \% \pm$ $3.59 \%, p<0.05$ ) (Fig. 2B-2D). The telomere length in the HG-siRNA group $(3.99 \pm 0.066)$ was longer than the HG group ( $3.03 \pm 0.096)$, but still shorter than control group (Fig. 3).

\section{The expression of $P_{53}$ and $P_{21}$}

We further determined the expression levels of $\mathrm{P}_{53}$ and P21. Results showed that $\mathrm{P}_{53}$ and $\mathrm{P}_{21}$ were highly expressed in GMCs grown under HG medium whereas $\mathrm{P}_{53}$ and $\mathrm{P}_{21}$ was barely detectable in the HG-siRNA group (Fig. 5).

\section{DISCUSSION}

Aging of kidney is associated with changes that are characteristic of somatic cell senescence in vitro, but the current mechanism is not clear. Oxidative stress plays a very important role in the occurrence and development of kidney aging. Therefore, in our study, HG was used to stimulate human GMCs. The results showed cell senescence phenotypes, including aging morphology with larger size and more cytoplasmic particles. SA- $\beta$-gal

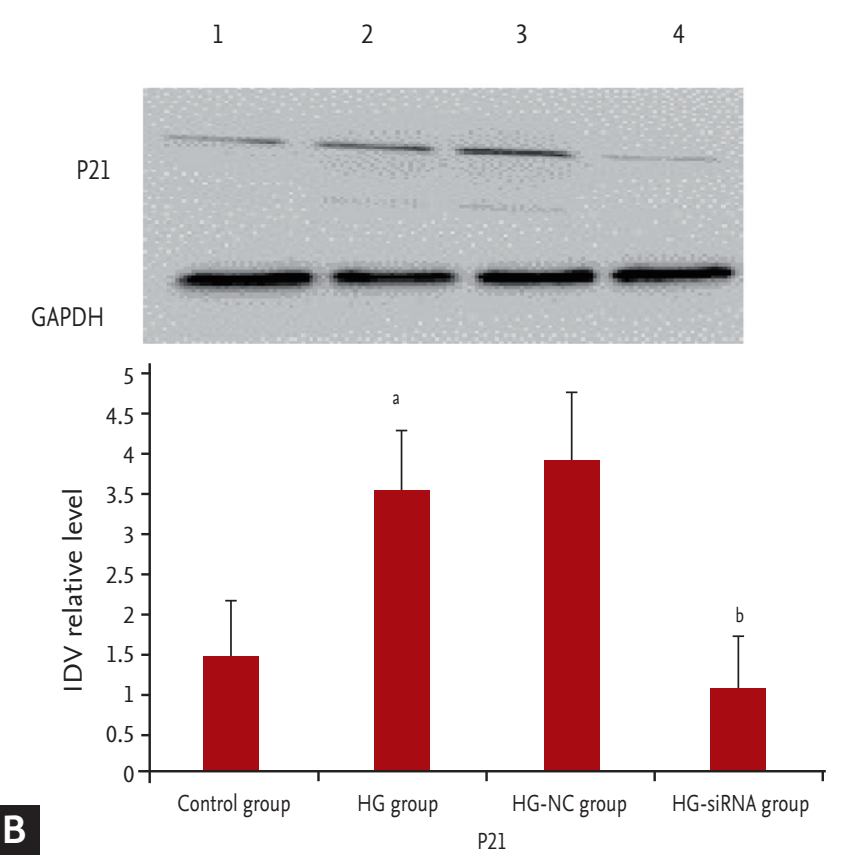

Figure 5. Western blot analysis. (A) Protein expression of P53. (B) Protein expression of P21. Lane 1, control group; lane 2, high glucose (HG)-group; lane 3, HG-negative control (NC) group; and lane 4, HG-siRNA group cells. Integrated density values (IDVs) of $\mathrm{P}_{53}$ are shown. GAPDH, glyceraldehyde 3-phosphate dehydrogenase. ${ }^{\mathrm{a}} \mathrm{p}<0.05$ vs. control group, ${ }^{\mathrm{b}} \mathrm{p}<0.05 \mathrm{vs}$. HG group. 
staining which is a reliable biomarker for cellular senescence was used to determine whether the replication arrest was associated with senescence. Compared with the control group, SA- $\beta$-gal staining was significantly increased by HG. These characteristics indicated that HG successfully induced GMCs senescence.

Telomeres are region of repetitive DNA at the end of a chromosome in eukaryotic cells. Telomeres form cap-like specialized structures and protect chromosome from digestion and degradation [10]. Each time a cell divides, the telomeres get progressive shorter, which lead to cell senescence [11]. Some environmental factors, including oxidative stress, inflammation, are associated with telomeres shortening acceleration [12,13]. In this study, we investigated the effect of HG on TRF lengths in GMCs by Southern blot analysis. HG-grown GMCs had lower TRF lengths than the control group. These results revealed that telomere DNA in GMCs lost significantly after 96 hours treatment with HG.

Caveolin-1 would be a prime candidate for such a role as a major determinant of the aging process. Caveolin-1 can associate with a variety of molecules, involved in signal transduction, endocytosis and transcytosis, cytoskeletal arrangement, etc. Recently, great progress has been made in the effect of caveolin-1 in stress-induced premature senescence. It has been reported that caveolin-1 expression is up-regulated in cultured human senescent fibroblasts and aging T-cells [14]. Subcytotoxic level of hydrogen peroxide up-regulates endogenous caveolin-1 expression and induces premature senescence in $\mathrm{NIH}$ 3T3 cells [15]. Bleomycin increases caveolin-1 expression and down-regulation of caveolin-1 expression by shRNA inhibits bleomycin-induced cell cycle arrest in A549 human lung adenocarcinoma epithelial cells [16].

It has been reported that caveolin-1 expression is up-regulated by cigarette smoking, a source of oxidative stress, and that caveolin-1 null mice were protected against cigarette smoke-induced premature senescence of lung fibroblasts and development of pulmonary emphysema. These data showed that caveolin-1 was involved in the process of accelerating cellular senescence by oxidative stress

Nothing is known about the presence of the caveolin-1 in the aging GMC. Therefore, we investigated the expression of caveolin-1 in aging GMCs, and found that the levels of caveolin-1 protein were increased in
GMCs after HG stimuli. We also analyzed alterations in cell aging after application of caveolin-1-siRNA. Optical microscopy revealed HG-siRNA group cells to be regularly aligned, with nuclear membrane invagination. Compared with the HG group, SA- $\beta$-gal staining was decreased in the HG-siRNA group. Accordingly, we further determined the change of telomere length after application of caveolin-1-siRNA. Results showed that compared with the HG group, telomere length was increased in the HG-siRNA group. These findings indicate that caveolin-1 plays a more important role in the senescence of GMCs, and blocking caveolin-1 can reduce cell senescence.

The specific mechanism of caveolin-1 involved in GMC cells aging is not clear. The senescence signals trigger a DNA damage response, and this response shares a common signaling pathway that converges on either or both of the well-established tumor suppressor protein pathways, $\mathrm{P}_{53} / \mathrm{P}_{21}$ and $\mathrm{P}_{16} / \mathrm{RB}$ [17]. It has been reported that caveolin-1 is involved in the senescence through $\mathrm{P}_{53} /$ P21 pathway. In an implication for evidence-based therapy of delayed wound healing in diabetes, caveolin-1/ polymerase I and transcript release factor (PTRF) upregulation constitutes a mechanism for mediating $\mathrm{P}_{53}$ induced cellular senescence [18]. After oxidative stress, caveolin-1 sequesters Mdm2 away from $\mathrm{P}_{53}$, leading to stabilization of $\mathrm{P}_{53}$ and up-regulation of $\mathrm{P}_{21}$ (Wafi/Cip1) in human fibroblasts. Expression of a peptide corresponding to the Mdm2 binding domain of caveolin-1 is sufficient to up-regulate $\mathrm{P}_{53}$ and $\mathrm{P}_{21}$ (Wafi/Cip1) protein expression and induce premature senescence. Oxidative stress-induced activation of the $\mathrm{P}_{53} / \mathrm{P}_{21}$ pathway and induction of premature senescence are compromised in caveolin-1 null mouse embryonic fibroblasts [19]. Accordingly, we further determined the expression levels of $\mathrm{P}_{53}$. Results showed that $\mathrm{P}_{53}$ was highly expressed in GMCs grown under HG medium whereas $\mathrm{P}_{53}$ was barely detectable in the HG-siRNA group. These results indicate that caveolin-1 regulates HG-induced senescence of GMCs through a novel P53-mediated pathway.

In summary, based on our observations, we confirmed that HG induced cell senescence in GMCs. The caveolin-1 is involved in HG-induced mesangial cell senescence, and blocking caveolin-1 significantly reduced cell senescence. The effect of caveolin-1 is mediated by $\mathrm{P}_{53}$ pathway. The results of our study, which are addressed 
HG-induced, may not be applicable to kidney senescence which is caused by other reasons. Further studies are required to understand.

\section{KEY MESSAGE}

1. The caveolin-1 is involved in high glucose (HG)-induced mesangial cell senescence, and blocking caveolin-1 significantly reduced cell senescence.

2. Caveolin-1 regulates HG-induced senescence of glomerular mesangial cells through a novel $\mathrm{P}_{53}$-mediated pathway.

\section{Conflict of interest}

No potential conflict of interest relevant to this article was reported.

\section{Acknowledgments}

This study was supported by a grant of the Science and technology Project of The First Affiliated Hospital of Liaoning Medical University, no. FYK201215. We thank for the research institute of The First Affiliated Hospital of Liaoning Medical University for valuable suggestions and help.

\section{REFERENCES}

1. Schmitt R, Cantley LG. The impact of aging on kidney repair. Am J Physiol Renal Physiol 2008;294:F1265-F1272.

2. Famulski KS, Halloran PF. Molecular events in kidney ageing. Curr Opin Nephrol Hypertens 2005;14:243-248.

3. Ben-Porath I, Weinberg RA. The signals and pathways activating cellular senescence. Int J Biochem Cell Biol 2005;37:961-976.

4. Itahana K, Campisi J, Dimri GP. Methods to detect biomarkers of cellular senescence: the senescence-associated beta-galactosidase assay. Methods Mol Biol 2007;371:21-31.

5. Zhou XJ, Saxena R, Liu Z, Vaziri ND, Silva FG. Renal senescence in 2008: progress and challenges. Int Urol Nephrol 2008;40:823-839.

6. Hackett TL, de Bruin HG, Shaheen F, et al. Caveolin-1 controls airway epithelial barrier function: implications for asthma. Am J Respir Cell Mol Biol 2013;49:662-671.

7. Parat MO, Riggins GJ. Caveolin-1, caveolae, and glioblastoma. Neuro Oncol 2012;14:679-688.

8. Minshall RD, Malik AB. Transport across the endothelium: regulation of endothelial permeability. Handb Exp Pharmacol 2006;(176 Pt 1):107-144.

9. Zou H, Stoppani E, Volonte D, Galbiati F. Caveolin-1, cellular senescence and age-related diseases. Mech Ageing Dev 2011;132:533-542.

10. Jiang H, Ju Z, Rudolph KL. Telomere shortening and ageing. Z Gerontol Geriatr 2007;40:314-324.

11. Percy C, Pat B, Poronnik P, Gobe G. Role of oxidative stress in age-associated chronic kidney pathologies. Adv Chronic Kidney Dis 2005;12:78-83.

12. Dabouras V, Rothermel A, Reininger-Mack A, Wien SL, Layer PG, Robitzki AA. Exogenous application of glucose induces aging in rat cerebral oligodendrocytes as revealed by alteration in telomere length. Neurosci Lett 2004;368:68-72.

13. Herbig U, Sedivy JM. Regulation of growth arrest in senescence: telomere damage is not the end of the story. Mech Ageing Dev 2006;127:16-24.

14. Volonte D, Galbiati F. Caveolin-1, cellular senescence and pulmonary emphysema. Aging (Albany NY) 2009;1:831835 .

15. Volonte D, Zhang K, Lisanti MP, Galbiati F. Expression of caveolin-1 induces premature cellular senescence in primary cultures of murine fibroblasts. Mol Biol Cell 2002;13:2502-2517.

16. Linge A, Weinhold K, Blasche R, Kasper M, Barth K. Downregulation of caveolin-1 affects bleomycin-induced growth arrest and cellular senescence in A549 cells. Int J Biochem Cell Biol 2007;39:1964-1974.

17. Jiang D, Attardi LD. Engaging the p53 metabolic brake drives senescence. Cell Res 2013;23:739-740.

18. Bitar MS, Abdel-Halim SM, Al-Mulla F. Caveolin-1/PTRF upregulation constitutes a mechanism for mediating p53-induced cellular senescence: implications for evidence-based therapy of delayed wound healing in diabetes. Am J Physiol Endocrinol Metab 2013;305:E951-E963.

19. Bartholomew JN, Volonte D, Galbiati F. Caveolin-1 regulates the antagonistic pleiotropic properties of cellular senescence through a novel Mdm2/p53-mediated pathway. Cancer Res 2009;69:2878-2886. 